\title{
The PMS Project: Poor Man's Supercomputer
}

\author{
F. Csikor ${ }^{1}$, Z. Fodor ${ }^{1}$, P. Hegedüs ${ }^{1}$, V.K. Horváth ${ }^{2}$, S.D. Katz ${ }^{1}$, A. Piróth ${ }^{1}$ \\ ${ }^{(1)}$ Institute for Theoretical Physics and ${ }^{(2)}$ Department of Biological Physics, \\ Eötvös University, Pázmány P. sétány 1/A, 1117 Budapest, Hungary
}

31 December, 1999

\begin{abstract}
We briefly describe the Poor Man's Supercomputer (PMS) project carried out at Eötvös University, Budapest. The goal was to construct a cost effective, scalable, fast parallel computer to perform numerical calculations of physical problems that can be implemented on a lattice with nearest neighbour interactions. To this end we developed the PMS architecture using PC components and designed a special, low cost communication hardware and the driver software for Linux OS. Our first implementation of PMS includes 32 nodes (PMS1). The performance of PMS1 was tested by Lattice Gauge Theory simulations. Using pure SU(3) gauge theory or the bosonic MSSM on PMS1 we obtained $3 \$ /$ Mflop and 0.45\$Mflop price-to-sustained performance ratio for double and single precision operations, respectively. The design of the special hardware and the communication driver are freely available upon request for non-profit organizations.
\end{abstract}

\section{Introduction}

Our purpose was to build a high performance supercomputer from PC elements. We use PCs for two reasons. They have excellent cost/performance ratios [1] and can easily be upgraded when faster motherboards and CPUs will be available. The PMS project started in 1998, and the machine is now ready for physical calculations. Our first PMS machine (PMS1) consists of 32 PCs arranged in a three-dimensional $2 \times 4 \times 4$ mesh. Each node has two special communication cards providing fast communication through flat cables to the six neighbours. This gives a much better performance than simple Ethernet link.

Since the machine is built from PCs, the latest versions of all programming languages (such as $\mathrm{C}$ and Fortran) can be used for coding. Writing applications is straightforward. One only has to keep in mind the 3-dimensional mesh structure of the machine; no further deep understanding of how the communication works is required. There are some routines written in $\mathrm{C}$ that make communication of data between the nodes easy. The machine works in Single Instruction Multiple Data (SIMD) mode: all processors execute the same program, while the data they work on may differ.

Nowadays double precision floating point arithmetic is necessary for accurate results. PMS offers this precision since the processors have double precision Floating Point Units (FPUs). In cases when single precision is enough, the special MMX instruction set of AMD K6-2 processors can be used. This provides a much higher performance, in principle 8 times higher, than the standard double precision mode.

The following sections describe the hardware and software architectures of PMS. We first give a short overview of the machine and then describe the hardware and the software in more detail. Some performance results are also presented, and an outlook is given. 


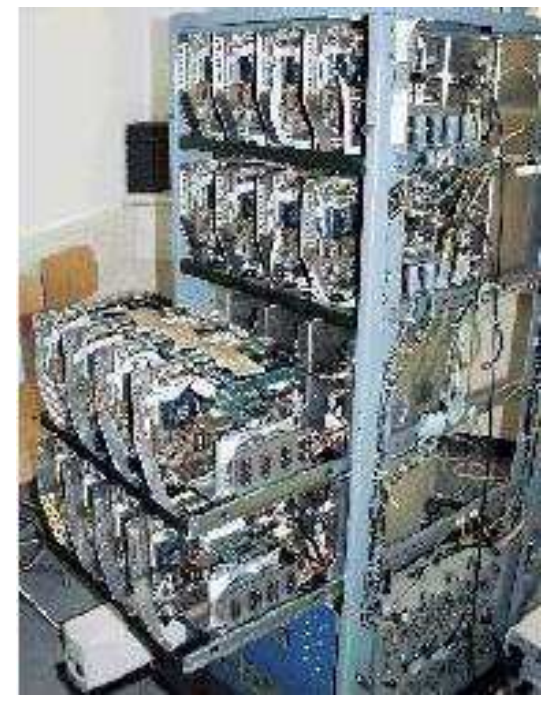

Figure 1: The PMS1 rack and the trays.

\section{Overview}

The nodes in PMS are based on PC components. In our first PMS machine (PMS1) the rack containes four trays (see Fig. 1). These trays hold eight nodes in two rows. Each node is powered by its own standard PC power supply located at the bottom of the rack. Appropriate air cooling by ventilation is provided for. Notice, that rack mounting is not required for the present communication speed of PMS1. Even PCs within their own cases can be used.

Each node in PMS is an almost complete PC. In PMS1 the configuration of a node consists of a $100 \mathrm{MHz}$ motherboard (SOYO SY-5EHM), a single 450MHz AMD K6-II processor, 128Mbyte (7nsec) SDRAM, 10Mbit Ethernet card, and a hard disk of caoacity 2.1 Gbyte.

The nominal speed of each node is 225 Mflop, since floating point operations of the AMD processor require two clock cycles. The price of one node was approximately $\$ 350$ (for the price of such a computer see [1]). The additional cost for the communication cards (see later) is $\$ 40$ for each node. So the price/performance ratio is reasonably low.

PMS uses special hardware for communication (PMS CH) to make high speed parallel calculations possible. The basic idea behind the hardware is that the PMS CH provides direct connection of each node to its nearest neighbours (NN). The PMS CH handles both polled port (PP) IO operations and direct memory access (DMA) between two selected nodes.

The PP IO mode is quite straightforward; the sender node sends a word and a flag indicating that a word is being sent. The sender holds the data until the receiver node sends an acknowledgement signal after reading the data. In case of the DMA the problem is synchronization between the sender and the receiver. Typical usage of DMA assumes data transfer between a PC and an another device. This device is usually either a slave or a bus master with appropriate circuits. If DMA is used to transfer data between two PCs then one has to deal with synchronization between two DMA chips. Both problems are solved by using First In First Out (FIFO) data buffers for read(receive) and write(send) operations and appropriate hardware hand-shaking circuits.

Prior to data transfer the direction of the data flow must be decided. Also, using DMA transfer receiving and sending procedures must be initiated in a synchronized fashion since long delays in DMA may lead to system crash. The PC architecture limits this synchronization to 2-30 bus clocks since both DMA and the interrupt have an invoking latency (which is not defined by the bus protocol and may depend on the chip-set applied on the motherboard).

Programming the PMS CH is a fairly simple task. It can be done under Linux and 32-bit 

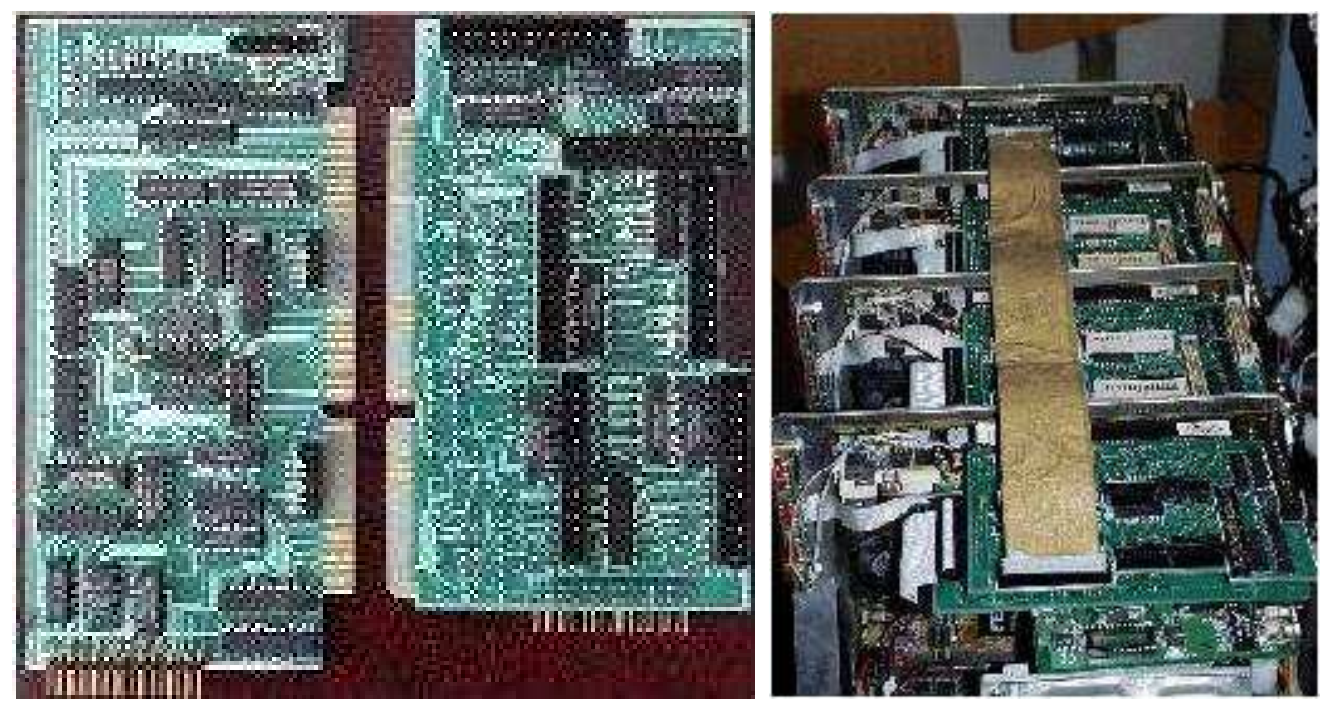

Figure 2: a, The PMS CPU and Relay cards. b, Nodes connected by flat cables in one direction.

(extended) DOS operating systems. All low-level device drivers are written in C and the programmer may use all kinds of commercial, share-ware or free-ware compilers. Using the communication drivers requires the knowledge of only a few functions. A simple programming example for Linux users can be found in the Appendix.

The PMS architecture does not define the bus used for the PMS CH. In PMS1 we have used the simplest ISA bus (see Fig. 2). Implementation of the PMS CH for other, faster buses is under development.

Our first implementation of the PMS CH includes two plug-in ISA cards, the PMS CPU card and the PMS Relay card. The nodes are arranged in a $2 \times 4 \times 4$ mesh as shown in Fig. 3. In each node both the PMS CPU and the PMS Relay cards are installed providing a fast communication to the six NNs. At the boundaries periodic boundary conditions are realized as indicated in Fig. 3 where the links at the boundaries correspond to the ones on the other sides. This determines the hardware architecture of the machine, which is similar to that of the APE machines [2].

Debian Linux 2.1 is installed on each node. After turning the power on each node boots from its own hard-disk. All nodes can be accessed through the Ethernet network. There is a main computer that controls the whole cluster. Some simple Linux scripts have been written to copy the executable program code and the appropriate data to and from the nodes and to execute the programs. Collecting the results is also managed by a simple Linux script. In principle, Ethernet connection could be used for data transfer between the nodes during simulations. However, this turns out to be too slow in most cases. One major reason for this is that any data transfer between two machines makes the whole network busy. Since building up the Ethernet connection is quite slow even for two computers, the Ethernet connection is not satisfactory. The theoretical maximum of the Ethernet connection speed is $1 \mathrm{Mbyte} / \mathrm{sec}$. This bottleneck could be avoided by using switch boxes, however efficient switch boxes are far too expensive and would dominate the price of the entire machine (cf. Fig. 6 and the corresponding discussion in the text below.)

The special communication cards -described in more detail in the next section- provide faster communication between adjacent nodes. However, this makes the machine applicable to local problems only, where communication only between the neighbouring nodes is necessary. In PMS1 the speed of communication through these cards -limited essentially by the ISA bus speed- is 2 Mbyte/sec, which is greater than the speed of our Ethernet by a factor of two. Furthermore to build up our communication practically no time is needed, while the build-up of the Ethernet connection is rather slow. Even more 16 pairs of machines can simultaneously communicate. Altogether we get two 


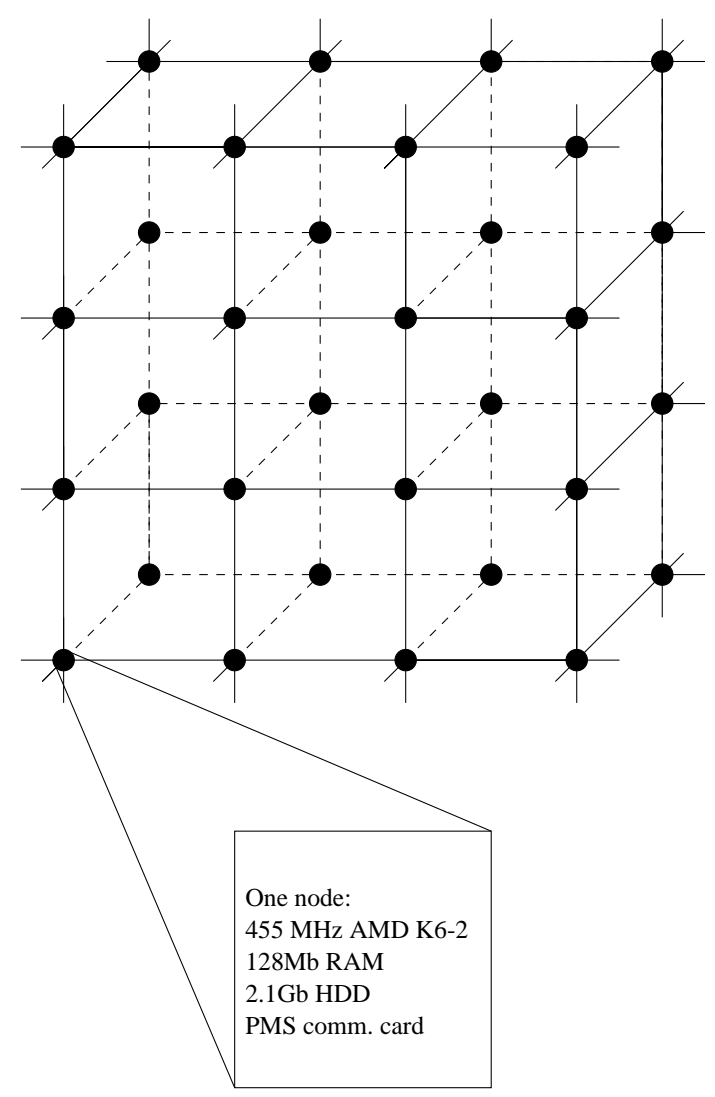

Figure 3: The PMS cluster

orders of magnitude better performance. Note that the system is scalable. One can build machines with larger number of nodes. The total inter-node communication performance is proportional to the number of nodes.

The whole computer is rack mounted. This way the flat cables needed for communication are relatively short. At current communication speeds this is not necessary. However, for future developments when communication gets much faster this can be crucial.

\section{Description of PMS Communication Hardware ( $\mathrm{CH})$}

There are two communication cards in each machine. The CPU card contains the main circuits needed for transmitting data, while the Relay card contains the connectors for the flat cables connecting the adjacent nodes and some additional circuits. The block diagram of the cards is shown in Fig. 4. The circuits of both cards are included. There are two 16-bit buffers, the output buffer and the input buffer, which accept the data coming from the computer and from one of the adjacent nodes, respectively. The Control Register is used -among other things- to clear the buffers and set the node to either sender or receiver state. The Cable Select circuit selects which direction the data is sent to or received from. The six directions are labelled as left (L), right (R), up (U), down (D), front (F), back (B). If the same physical cable is selected by two adjacent nodes, one of them set as sender and the other as receiver, a physical connection is established and the content of the output register of the sender is immediately transferred to the receiver's input register. The Local State Indicator (LSI) and the Remote State Indicator (RSI) are two registers to indicate the states of the nodes. There are 12 LSI and 12 RSI lines. They correspond to sending to and receiving from the six directions. Each node can indicate its request for sending or receiving through the LSI lines. The RSI lines are identical to the six neighbours' LSI lines. If there is a match between RSI and LSI 


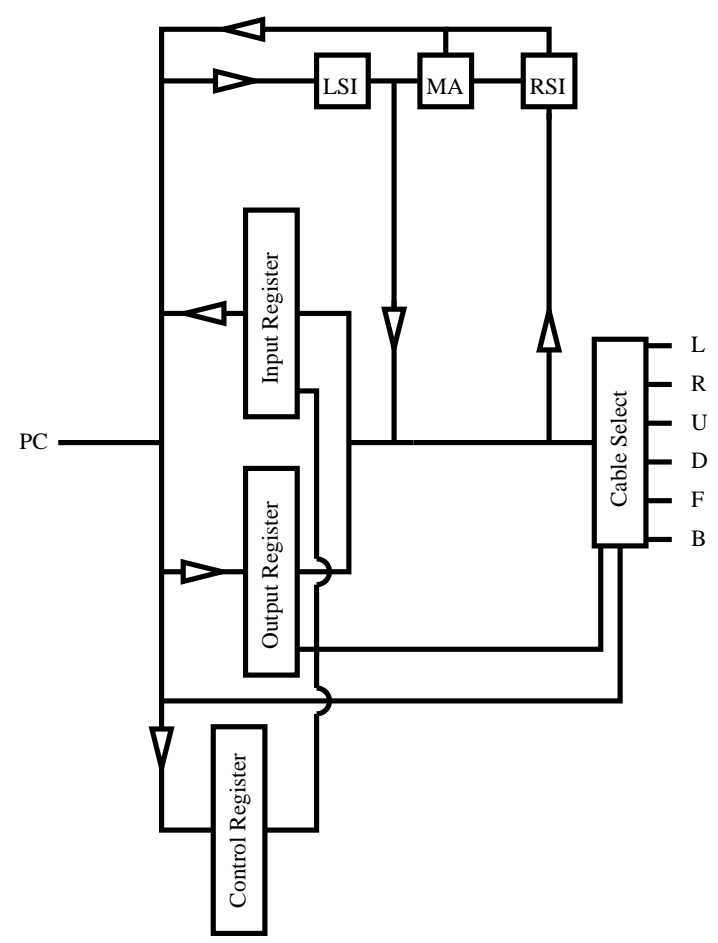

Figure 4: The PMS communication card

signals (i.e. a send and a corresponding receive request coincide) then the Match Any (MA) bit is set and an IRQ is generated on the PC bus. The interrupt is generated on both nodes at the same time, so the interrupt handlers on both machines can safely start transferring data without any extra syncronization. The data can be transferred either by DMA or by consecutive I/O operations.

The Cable Select circuit, the LSI, RSI and MA registers together with the flat cable connectors are located on the relay card, while all other circuits are located on the CPU card.

\section{Software}

As mentioned above, the whole cluster is connected via an Ethernet network. Each node has a special hostname that corresponds to its location in the cluster. The hostnames are s000,s001,s002,s003,s010 ... s133. The numbers in the hostname correspond to the coordinates of the node in the three dimensional mesh. When it is necessary for a node to identify itself (e.g. write/read priorities during inter-node communication, see below) the file '/etc/hostname' is used. The Ethernet network is used only for job management, i.e. to distribute and collect data to and from the nodes. It is not used for inter-node communication during simulations. This is achieved by the special hardware described in the previous section.

In order to take advantage of the fast communication from applications (e.g. high level $\mathrm{C}, \mathrm{C}++$ or Fortran code), a low-level Linux kernel driver has been developed to access all the registers of the communication card. From the user level cards can simply be reached by reading or writing the device files '/dev/pms0, /dev/pms1 ... / / dev/pms5'. The six device files correspond to the six directions. A write operation to one of these device files will transmit data to the corresponding direction, and reading from these device files reads out previously transferred data. All the necessary input/output operations for transferring data blocks are performed by the device driver. Notice the important feature that this can be reached from any high level $\mathrm{C}, \mathrm{C}++$, Fortran code for which compilers are available.

The main structure of the device driver is similar to that of the card. There are six read buffers, 
one for each direction in the main memory of the machine and there is one write buffer. The data are always written to the write buffer and read out from one of the read buffers.

The driver has two main parts. The first part is accessed from applications when the user writes or reads any of the device files '/dev/pms*'. The other part is the interrupt handler where the real data transfer takes place.

Whenever data are written to one of the device files, all the driver does is to copy the written data to the write buffer and set the corresponding LSI send signal to indicate that a data send is requested. If the buffer is already full, an error byte is returned to the application. Reading from the device files is similar: if there are data in the corresponding read buffer, they are sent to the application and the LSI receive line is set, since the node is ready to receive new data. If the read buffer is empty, an 'End Of File' byte is returned to the application.

When there is a coincidence between corresponding LSI and RSI signals, an interrupt is generated by the card, which invokes the driver's interrupt handler. It is the task of the handler to transfer data from the sender to the receiver. The interrupt handlers on the two communicating nodes start almost at the same time. The difference may only be a few clock cycles. There is, however, a need for synchronization. If the machines are ready to send or receive the first byte they indicate it with their LSI lines. Notice that this will not cause an extra interrupt since interrupts are disabled within the interrupt handler. The sender first transmits the size of the package that will follow in 16-bit words. In the present version this is a 16-bit value, so the maximum size of a package that can be transferred is 128 kbytes. Then the given number of 16-bit values follow. Each word from the sender's write buffer is copied to the receiver's read buffer. Finally, a 32-bit checksum is sent. The receiver computes its own checksum and if it does not match the received checksum, it is indicated to the sender and the whole transfer is repeated. The final step in the interrupt handler is to clear the LSI lines of both nodes. On the one hand this indicates for the sender that the data have been transferred and the write buffer is empty again. On the other hand this tells the receiver that the corresponding read buffer is full, so no new data can arrive unless the buffer is emptied.

The buffer sizes are set in the driver to constant values. From the previous paragraph it is clear that the maximum reasonable buffer size is 128 kbytes. In order to save memory, while allowing large packages at the same time the buffer size is set to only 64 kbytes at present.

The driver makes application programming quite easy. Communication can be achieved by accessing the above mentioned device files. However, some $\mathrm{C}$ functions have also been written to make writing applications even simpler. These functions are the following:

pms_open is used to initialize the card. It clears all buffers, sets the LSI receive lines, clears the LSI send lines and enables interrupts. The node thus becomes ready to receive data from any of the neighbours.

pms_close is used to close the card. All LSI lines are cleared and interrupts are disabled. No further communication may take place after this function call.

pms_send, pms_recv are used to send and receive data. Their parameters are the direction, the number of bytes to send, and a pointer to the beginning of the data. On success they return a positive value, otherwise a negative one. If there is no data in the read buffer, pms_recv returns 0 .

The driver does not take care of any priority problems. It is possible to write applications that will not work since all nodes are waiting for data while none of them is sending anything. This is often the case when the same code is running on all the nodes without any priority check. There is a simple solution to these kind of problems. The parity of the node is simply the parity of the sum of the three digits in its hostname. Each time when communication is performed, even nodes send data first and receive afterwards, while odd nodes receive first and send their data afterwards. This simple method works in most cases. Note that the code for it is located in the application $(\mathrm{C}, \mathrm{C}++$ or Fortran) and not in the driver. 


\section{Performance}

We carried out lattice gauge theory simulations on our parallel computer PMS. We used double precision variables. Two types of theories were studied.

a. We analyzed the pure $\mathrm{SU}(3)$ gauge theory with the simplest Wilson action. Overrelaxation and heatbath updating algorithms were used for the link variables. The most CPU time consuming part, manipulation with $3 \times 3$ matrices, was written in assembly language. This increased the speed of the code by more than a factor of two.

b. We studied the bosonic sector of the minimal supersymmetric extension of the standard model (MSSM). SU(2) and SU(3) gauge fields were included with two complex Higgs doublets and with left and right handed coloured scalar quarks. Again, overrelaxation and heatbath updating algorithms were used for both gauge link variables and scalar site variables. The microcanonical overrelaxation update for scalar quarks is quite complicated and new, its details will be discussed elsewhere [3]. Manipulation with $3 \times 3$ and $2 \times 2$ matrices was written in assembly language, which again increased the speed of the code essentially.

Having carried out lattice simulations for these two theories we obtained similar results for the speed of the code and for the communication between nodes. The MSSM results for communication are actually somewhat better. The reason for that is quite simple. The number of variables in the MSSM is larger by a factor of two than in pure SU(3) gauge theory; however, the number of floating point operations needed for a full update is more than an order of magnitude larger. Thus, for the same lattice size the time needed to transfer the surface variables -done by the communication cardscompared to the update time is smaller for MSSM than for the pure SU(3) theory. We expect that a similar though less pronounced effect should be observed for fermionic systems e.g. with multiboson [5] algorithms. Since it is much more straightforward to compare our SU(3) results than those of the MSSM simulations with the results of the literature, in the following paragraphs we discuss the $\mathrm{SU}(3)$ case, too.

For small lattice sizes the most economical way to use our $32 \mathrm{PC}$ cluster is to put independent lattices on the different nodes. The maximum lattice size in the $\mathrm{SU}(3)$ theory for 128 Mbyte memory is $\sim 20^{4}$, or for finite temperature systems $6 \cdot 32^{3}$. One thermalizes such a system on a single node, then distributes the configuration to the other nodes and continues the updating on all 32 nodes. We measured the sustained performance of the cluster in this case, which gave $32 \times 152 \mathrm{Mflop}=4.9 \mathrm{Gflop}$. This $152 \mathrm{Mflop} /$ node performance means that one double precision operation is carried out practically for every third clock cycle of the $450 \mathrm{MHz}$, whereas the nominal maximum of the processor is one operation for every second clock cycle. As it was mentioned above, without assembly programming an approximate reduction factor of two in the performance was observed.

Increasing the volume of the simulated system one can divide the lattice between 2 nodes (the $2 \times 4 \times 4$ topology has 2 nodes in one of the directions). For even larger lattices one can use 4 nodes ( 4 in one direction), 8 nodes $(2 \times 4$ in two directions) 16 nodes $(4 \times 4$ in two directions) or 32 nodes $(2 \times 4 \times 4$ in three directions). Again, the most economical way to perform the simulations is to prepare one thermalized configuration and put it on other nodes (this method obviously can not be used for the $2 \times 4 \times 4$ topology, because in this case the whole machine with 32 nodes is just one lattice).

Based on our measurements we determined the sustained performance of a 32-node PMS cluster as a function of the lattice volume. The result for a set of lattice volumes for finite temperature systems with temporal extension, $L_{t}=6$ for $\mathrm{SU}(3)$ and $L_{t}=4$ for MSSM can be seen on Fig. 5. Clearly, the largest volume one can reach is approximately twice as large for the SU(3) gauge theory than for the MSSM. For both cases there are regions where the performance increases with the volume. This can be easily explained by the fact that larger volume means better surface/volume ratio, thus better performance. There are three drops in the performance for both SU(3) and MSSM. 


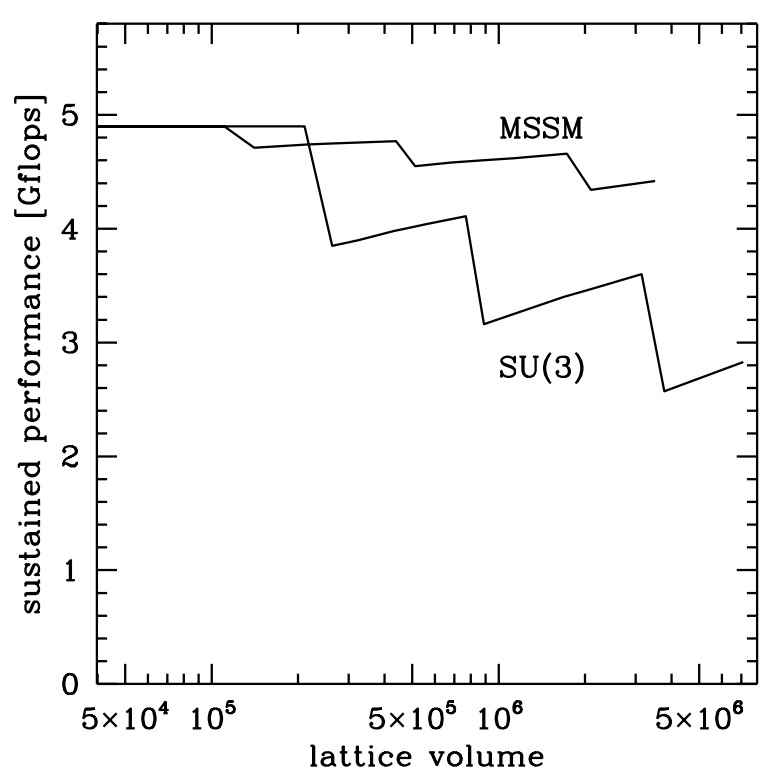

Figure 5: Sustained performance of PMS as a function of the lattice volume for pure SU(3) gauge theory and for MSSM. The endpoints of the lines correspond to the largest volumes which can be simulated on a $32 \mathrm{PC}$ cluster.

They correspond to lattice volumes for which new communication directions were opened (or, in other words, the dimension of the mesh of the nodes on which the lattice was divided, increased by one) in order to fit the lattice into the available RAMs. As it can be seen the performance for the MSSM is still very high even at the largest volume with three-dimensional communication: it is just $10 \%$ smaller than the performance without communication. This plot gives us the optimum architecture of such a parallel computer. The number of nodes and the number of communication directions used for a given lattice should be as small as possible simultaneously. This means a $2 \times 4 \times 4$ topology for 32 nodes and a $2 \times 4 \times 8$ topology 64 nodes.

Despite the fact that the speed of the communication between two nodes is not that high $(2$ Mbit/sec) the performance of the cluster is quite good. The reason for this is the high speed of the individual nodes $(450 \mathrm{MHz})$ and the large RAM on each node. This sort of design does not need a division of the lattice to hundreds of sub-lattices, thus it does not need a very fast communication. Clearly, the use of PCI communication instead of ISA gives an order of magnitude faster communication, which increases the potential of such a machine.

In order to compare our machine with other existing (and proposed) parallel systems used in lattice gauge theory, we use the plot of N. Christ [4]. As it can be seen in Fig. 6, PMS1 is perhaps the best existing machine as far as price/sustained performance is concerned. It is particularly important to emphasize that the presented performance $3 \$$ Mflop with $\approx 4$ Gflop total performance of PMS1 is calculated for double precision operations (while some of the performances of the machines on the plot are given for single precision operations). For single precision simulations one can use the MMX instruction set which is 8 times faster than the double precision operations (4 operations for each clock cycle). We estimated the single precision performance by assuming that MMX programming results in $20 \%$ decrease in performance. The total performance of PMS1 is $\approx 27$ Gflop with $0.45 \$ /$ Mflop priceto-sustained performance ratio. (Notice that our measured performance is based on double precision code, whereas single precision numbers are estimated ones.) Our PMS1 machine, similarly to other workstation farms has a moderate maximum sustained performance as compared to Teraflop-scale machines (CP-PACS [6] or QCDSP [7]). However, PMS1 has a much better price/performance ratio than other workstation farms. The reason for this is threefold. 


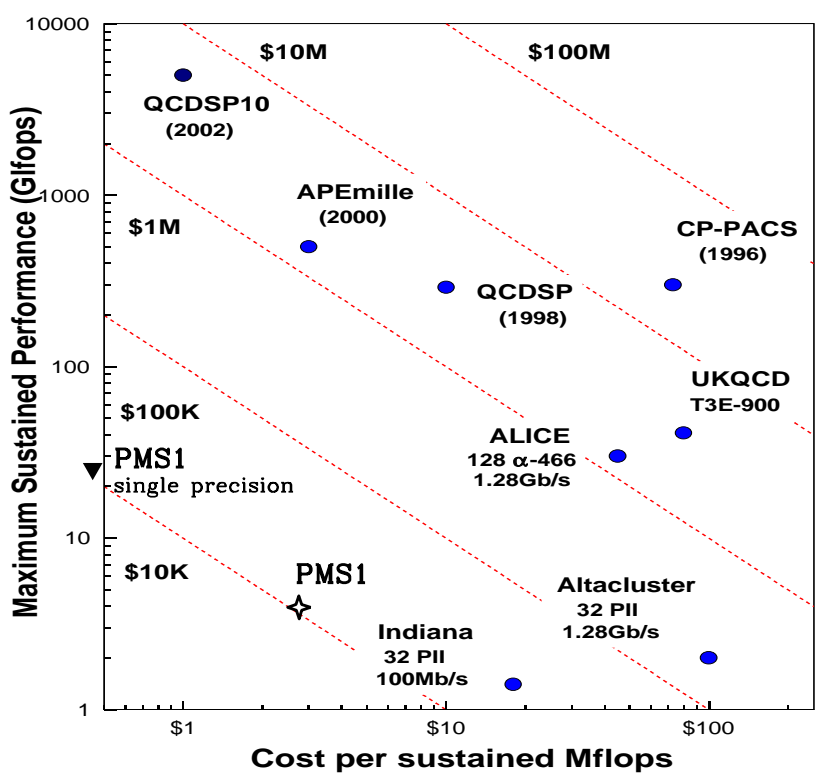

Figure 6: Sustained performance of different machines used in lattice gauge theory as a function of price/performance. Our PMS machine is presented as a $32 \mathrm{PC}$ based system.

a. At the day of purchase our choice for the PC components was optimized for price/performance. b. Machines with 100Mbit fast Ethernet (e.g. Indiana) are inexpensive, but communication is slow, resulting in a high price/performance ratio.

c. Machines with much faster networks using Myrinet (e.g. Alice [8] and Altacluster) give higher performance. However, this is balanced in the price/performance ratio by the higher cost of the network.

The essential feature of PMS architecture is that the speed of the communication (depending on the size of the machine) is comparable to that of Myrinet. However, the price for such a communication is as low as $\approx 40 \$ /$ node, which is more than an order of magnitude less than the cost of Myrinet.

\section{Conclusion}

We have presented a description of the status of PMS project. Details of the communication hardware and software have been explained. The existing 32-node machine (PMS1) is able to perform medium size LGT simulations in case of nearest neighbour interactions, distributing the lattice on several nodes, if necessary. This is done at a very good price/sustained performance ratio. Problems requiring more number crunching and less communication give a better performance.

We plan to increase the number of connected nodes and implement the PMS CH for faster buses in order to increase the maximum size of programs and enhance communication speed.

Finally, we would like to emphasize that the design of our communication hardware and the developed Linux drivers are freely available for non-profit organizations. Those who intend to build a PMS architecture machine have simply to buy PCs produce the communication cards and connect the nodes by flat cables. Furthermore they have to install Linux and our Linux driver. Questions and requests for information should be sent to pms@labor.elte.hu. 


\section{Acknowledgement}

This work was supported by Hungarian Science Foundation Grants under Contract Nos. OTKAT22929-T29803-F17310-M28413/FKFP-0128/1997.

\section{Appendix}

The following $\mathrm{C}$ code is a simple example to illustrate the usage of PMS communication cards in a given application. This example transfers random numbers in each direction. It can be used to test all channels. We performed this test on all cards by sending 100 Gbytes of data on each channel. We observed no errors. The header file 'pms.h' that contains the function prototypes is also included.

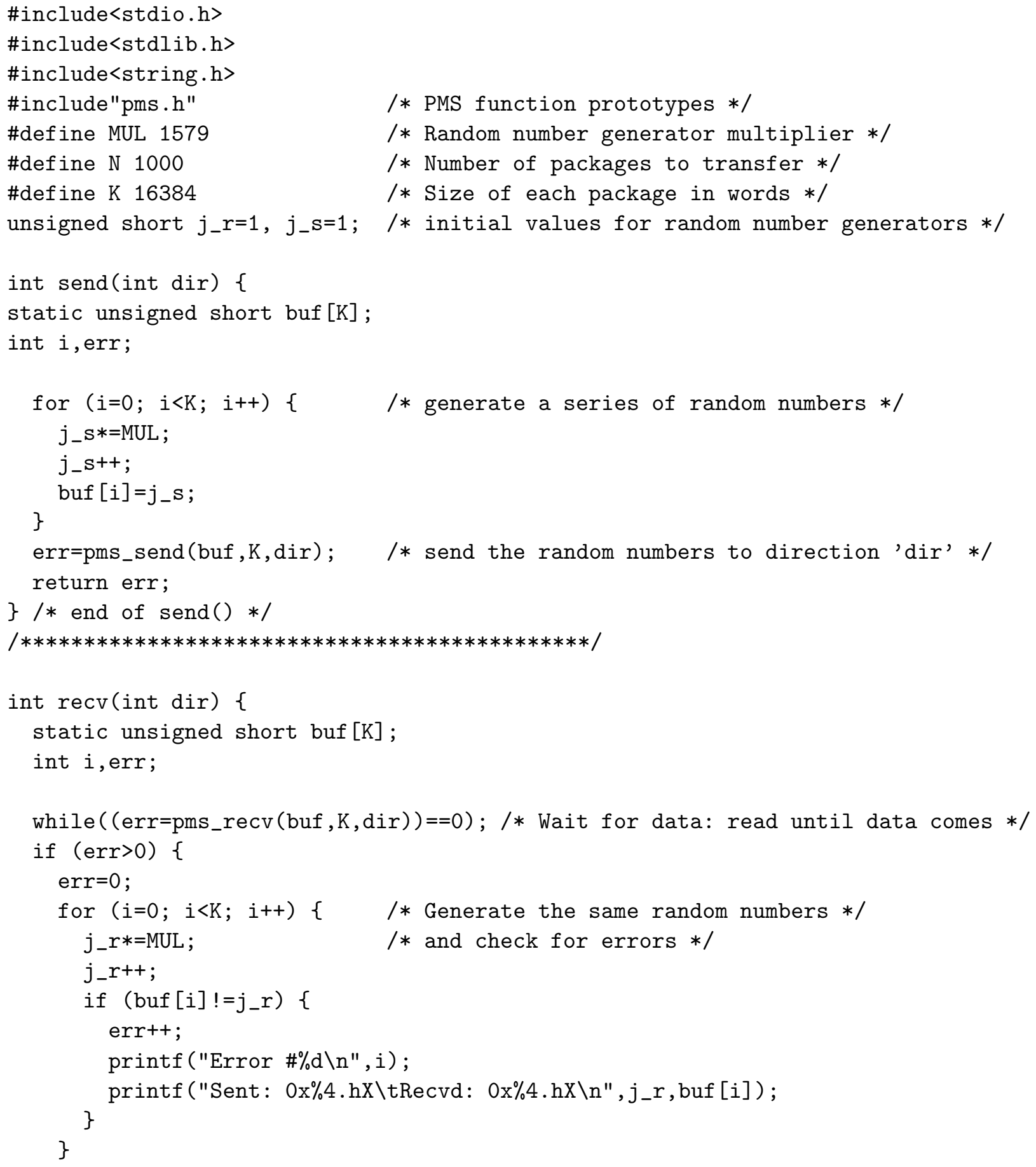


\}

return err;

\}/* end of $\operatorname{recv}() *$ /

$/ * * * * * * * * * * * * * * * * * * * * * * * * * * * * * * * * * * * * * * * * * * * * * * * /$

void main(int argc, char * argv[]) \{

int $i$;

FILE * hostname;

char host [10];

int pos [3], par;

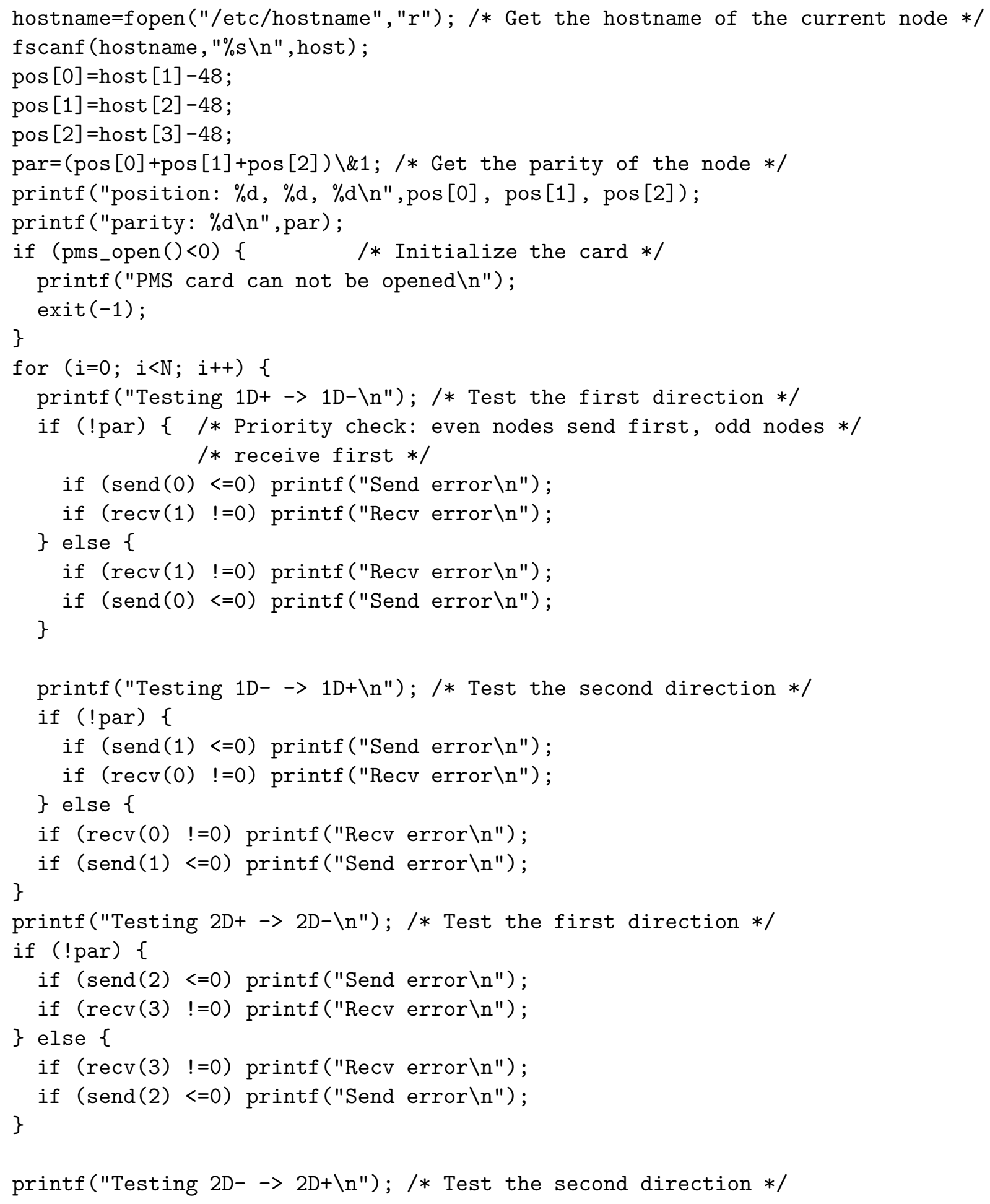




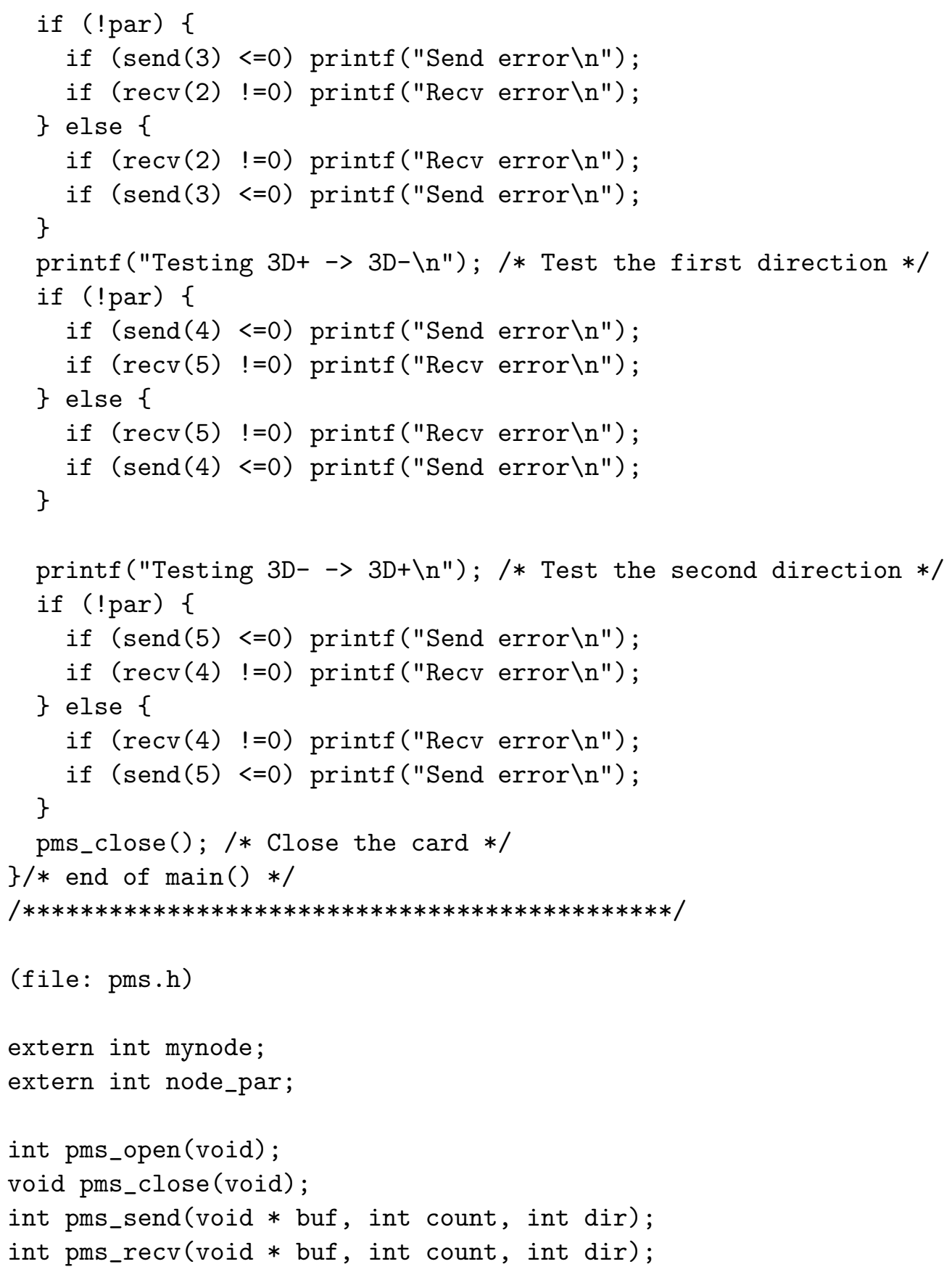

\section{References}

[1] http://www.pricewatch.com

[2] Comput. Phys. Commun. 57 (1989) 285; A. Bartonoli et al., Nucl. Phys. B (Proc. Supl.) 60A (1998) 237; http://chimera.roma1.infn.it/ape.htm]; F. Aglietti et al., Nucl. Instrum. Meth. A389 (1997) 56.

[3] F. Csikor et al., in preparation.

[4] N. Christ, hep-lat/9912009.

[5] M. Lüscher, Nucl. Phys. B418 (1994) 637; I. Campos et al, Eur. Phys. J. C11 (1999) 507; Ph. de Forcrand, hep-lat/9903035. 
[6] Y. Iwasaki, Nucl. Phys. (Proc. Suppl.) 60A (1998) 246; S. Aoki et al., hep-lat/9903001; http://www.rccp.tsukuba.ac.jp.

[7] D. Chen et al., Nucl. Phys. B (Proc. Suppl.) 73 (1999) 898; http://phys.columbia.edu/ cqft.

[8] N. Eicker et al., hep-lat/9909146. 